\title{
Estudio exploratorio de las interacciones musicales entre hermanos/as en el entorno cotidiano
}

\section{Exploratory study of the musical interactions between siblings in the everyday setting}

\author{
Marta Dosaiguas Canal \\ Marta.dosaiguas@uab.cat \\ Departamento de Didáctica de la Expresión Musical, Plástica y Corpora \\ Universidad Autónoma de Barcelona \\ Barcelona, España \\ ORCID: https://orcid.org/0000-0002-3343-2569
}

Jèssica Pérez-Moreno

Jessica.perez@uab.cat

Departamento de Didáctica de la Expresión Musical, Plástica y Corporal Universidad Autónoma de Barcelona

Barcelona, España

ORCID: https://orcid.org/0000-0002-1685-8859

doi: 10.7203/LEEME.48.21417

Recibido: 31-07-2021 Aceptado: 19-11-2021. Contacto y correspondencia: Marta Dosaiguas Canal, Departamento de Didáctica de la Expresión Musical, Plástica y Corporal, Universidad Autónoma de Barcelona, Campus UAB, C.P. 08193 Barcelona. España.

\section{Resumen}

Es evidente que en el seno familiar se producen interacciones musicales entre hermanos/as y que estas tienen una transcendencia importante en el desarrollo musical de ambos participantes. Este artículo presenta datos de un estudio sobre estas interacciones de las que aún se sabe muy poco. Los/as participantes son hermanos/as de entre dos y seis años de tres familias catalanas de características similares. Los datos se recogen mediante el DLP (Digital Language Processor) de LENA®, una grabadora de audio que puede grabar hasta 16 horas con una alta calidad y de forma no intrusiva, que lleva puesta el hermano menor. Las grabaciones se recogen de forma periódica y durante un día entero, y se completan con notas de voz narradas por las familias para facilitar información del contexto de ese día. Los datos se analizan con una tabla validada que permite extraer información de cuatro dimensiones: 1) orden de participación; 2) lugar; 3) tipo de intervención, y 4) fuente. Los resultados revelan, entre otra información, que: a) en la mayor parte de las interacciones el hermano mayor empieza y el menor termina la interacción; b) que las interacciones se producen mayoritariamente en el hogar; c) que la imitación y la sincronía son las tipologías de interacción más utilizadas, y d) que las interacciones se basan por igual en canciones o en improvisaciones.

Palabras clave: Educación Infantil; educación musical; educación en casa y en la vida familiar; hermanos/as.

\section{Abstract}

It is obvious that musical interactions between siblings take place within the family environment and are significant for their musical development. This paper presents data from a study on these interactions about which even now very little is known. Participants are siblings from two to six years old from three Catalan families with similar characteristics. Data is collected by LENA®'s DLP (Digital Language Processor) tool, an audio recorder capable of recording for 16 hours, with high quality and in a non-intrusive way, that is worn by the younger sibling. Recordings were collected periodically and for a whole day and were complemented with some voice messages narrated by the parents to contextualize the recording day. Data has been analysed using a validated table to extract information about four dimensions: 1) participation order, 2) place, 3) interaction type, and 4) source. Results reveal, among other information, that: a) in most of the interactions the older sibling starts the musical episode and the younger one finishes it, b) the interactions happen mostly at home; c) imitation and synchrony are very used, and d) interactions are based equally on songs and improvisations.

Key words: Early Childhood Education; Music Education; Home and Family Life Education; Siblings. 


\section{Introducción}

Según el estudio de Dunn (2007), el $80 \%$ de la población de los Estados Unidos y de Europa tiene como mínimo un/a hermano/a. La relación con este suele perdurar en el tiempo y, por eso, existen estudios sobre las relaciones entre hermanos/as a lo largo de la vida (Dunn, 2007; Connidis y Campbell, 1995; Eriksen y Gerstel, 2002; Weaver, Coleman y Ganong, 2003; Spitze y Trent, 2018). Tal y como ya apuntaba Vigotsky (1978), aprendemos a partir de los miembros más expertos de la cultura; dado que el vínculo fraternal es muy estrecho, en contraste con las relaciones con los progenitores, los/as hermanos/as actúan a un mismo nivel: se comparan entre sí, median, compiten por el amor y aceptación parental y desempeñan un papel determinante en la aplicación de esta teoría del aprendizaje en el contexto familiar.

Antes de la década de 1970, las interacciones entre niños se entendían en comparación con las interacciones de los adultos (Koops y Kuebel, 2019). Piaget (1965) ya había descrito las interacciones como recíprocas (de niño a niño) o complementarias (de niño a adulto), pero hasta años después no se empezó a identificar este último tipo en la comunicación entre niños y, especialmente, entre hermanos. Por ejemplo, Teti (2001) definió la interacción complementaria como "cualquier relación entre dos individuos que difiere en el desarrollo de niveles y competencias" (p.195).

Las relaciones entre hermanos tienen dos características específicas que ocasionan, como consecuencia, el establecimiento de unas relaciones estrechas (Dunn, 2007). Por un lado, está la calidad emocional: los/as hermanos/as pueden tener sentimientos intensamente positivos e intensamente negativos entre sí de forma frecuente y sin inhibiciones. Por otro lado, la familiaridad e intimidad de la relación intensifica los vínculos en comparación con otras relaciones. Desde la etapa infantil y hasta media infancia, pasan más tiempo juntos e interaccionando que con ninguna otra persona, incluidos los progenitores (McHale y Crouter, 1996 [en Dunn, 2007, p.310]). Como puede verse, las relaciones entre hermanos/as son ricas y variadas. No obstante, los/as hermanos/as mayores suelen mostrar una sensibilidad cognitiva que permite ofrecer al menor un contexto rico en interacciones multimodales, entendidas como intercambios basados en diferentes modos comunicativos (Norris, 2004). A su vez, promueven el desarrollo del lenguaje de los más pequeños (Prime et al., 2014). Como se sabe, el desarrollo musical y el del lenguaje en edad infantil están íntimamente relacionados (Pérez-Moreno, 2017; Brandt et al., 2012).

El objetivo de esta investigación es conocer las características musicales y específicas de las interacciones musicales entre hermanos/as en el entorno cotidiano con la finalidad de visibilizarlas como actividad cotidiana desarrollada en contexto no formal. La concepción musical en la que se basa está inmersa en la teoría de Blacking (2006), que define la música como un artefacto de "sonido humanamente organizado" (p.38). Más específicamente y en la línea de 
Casals (2017), se comparte la definición de música como "lenguaje formado por sonidos y silencios organizados humana y socialmente de una forma particular en cada cultura, y con unos significados, valores y sentimientos asociados" (p.28). En el presente estudio, se han analizado los sonidos organizados que provocan una respuesta sonora y organizada del hermano/a, por lo que se produce un intercambio sonoro y musical al que se ha denominado "interacción musical". Se han analizado fragmentos calificados como musicales por el uso concreto de las alturas y ritmos utilizados por los pequeños, que distan de la expresión hablada, sabiendo que a veces el uso de la lengua puede ser muy musical y es difícil saber dónde está la línea entre la declamación (lenguaje hablado) y la entonación (lenguaje cantado) (Bordons y Casals, 2012).

\subsection{Interacciones musicales entre hermanas y hermanos}

En lo concerniente a las interacciones entre hermanos/as, en el ámbito musical existen referencias a estas en las descripciones de ambientes musicales o interacciones domésticas en estudios en contextos familiares (Barrett, 2009; Barrett y Stauffer, 2012; Benetti, 2017; Forrester, 2010; Gingras, 2012; Wu, 2018), sin ser dicha relación el foco de interés de la investigación. Según Cirelli et al. (2020), los/as hermanos/as mayores marcan los pasos y dan mayores oportunidades para el desarrollo musical de los pequeños, que se benefician de la mayor adquisición de habilidades musicales del mayor. A su vez, la familia da más oportunidades a los mayores para enseñar, guiar y cuidar a los pequeños (Howe et al., 2014). Howe et al. (2016) reafirman que las relaciones entre enseñar y aprender en los/as hermanos/as dependen de quién es el experto en el tema, no de su edad.

Un ejemplo sería el descrito en el estudio de Barrett (2009), quien observó cómo la hermana mayor, de cuatro años, adoptaba el rol de padre/madre con su hermano de dos, iniciando así interacciones musicales con él. Sole (2014) estudió la interacción de dos mellizos en los momentos previos al descanso nocturno. Como parte de los datos de los mellizos, describe que la interacción se realiza de manera desigual, ya que es la hermana quien mayoritariamente empieza a cantar y el hermano quien la sigue. Costa-Giomi y Sun (2016) estudian la interacción entre los miembros de una familia con dos gemelos de 15 meses y un hermano de tres años, donde el mayor ocupa el rol de experto. Recientemente, un estudio sobre las interacciones entre hermanos durante un día confirma que la hermana mayor es capaz de calmar al hermano menor y cantarle un repertorio específico, asumiendo un rol de cuidadora (Dosaiguas et al., 2021).

\subsection{La recogida de datos en estudios sobre educación musical en el contexto familiar}

Tradicionalmente, escoger el instrumento de recogida de datos de las investigaciones en contactos familiares ha supuesto un hándicap (Dosaiguas, 2021). Sabemos que en el contexto familiar se producen muchos aprendizajes, pero la dificultad de acceso a este entorno ha encaminado a la mayoría de los estudios sobre educación musical hacia los contextos formales, 
mucho más concretos en el espacio y el tiempo. Algunos investigadores-progenitores han utilizado su rol de progenitor para extraer datos sobre la educación en contextos no formales (Dean, 2011; de Vries, 2006; Forrester, 2010; Papoušek y Papoušek, 1981), pero coinciden en que la proximidad con los datos puede poner en riesgo la objetividad del análisis.

En las pocas investigaciones naturalistas existentes - en el sentido de que intentan respetar el medio donde se producen de forma habitual - en educación musical en la etapa infantil, se ha visto que el aprendizaje se produce con mucha frecuencia y en muchas direcciones: entre padres e hijos, pero también entre hermanos/as, amistades o personas próximas (Brodsky et al., 2020; Costa-Giomi y Sun, 2016; Dean, 2019; Koops, 2020; Politimou et al., 2018; Schaal et al., 2020; Vilar, 2014; Young, 2016). Campbell (2010) recuerda que la enculturación musical nos afecta desde antes de nacer y que no solo el ambiente familiar, sino todas las influencias culturales nos afectan; con lo cual, existen muchas oportunidades de aprender música: "los individuos adquieren la competencia cultural por ósmosis, absorbiendo las diversas facetas de su ambiente en el hogar, aprendiendo por el hecho de vivir en una familia, comunidad o cultura" [individuals achieve cultural competence by way of osmosis, absorbing the many facets of their home environment, learning by virtue of living within a family, community, or culture] (p.66).

\section{Método}

Este estudio de carácter exploratorio se enmarca en métodos de investigación cualitativos. Desde el paradigma interpretativo, se busca la comprensión del entorno cotidiano, aceptando la existencia de perspectivas múltiples y abiertas a cambios (Willis, 2007), para poder entender los procesos sociales de forma rica y profunda (Thanh y Thanh, 2015).

\subsection{Contexto}

Tal y como se ha podido comprobar en el marco teórico, existe una escasez de trabajos sobre la interacción musical fraternal. Esta investigación se enmarca en un contexto de educación no formal, en el seno de tres familias con dos hermanos/as, con el propósito de hallar sus interacciones musicales.

Fue en el seminario de la Early Childhood Music Commission de la International Society for Music Education que se celebró en Israel en verano de 2018 cuando la Dra. Costa-Giomi compartió con los asistentes el uso de las grabadoras DLP de LENA® en la investigación en educación musical. Esta herramienta despertó mucho interés entre todos los asistentes, que tuvieron ocasión de conversar sobre los frentes en los que sería interesante su utilización. Los datos del presente estudio se obtuvieron con esta grabadora de audio, discreta y de larga duración, utilizada en estudios previos sobre educación musical (Costa-Giomi y Benetti, 2017; Costa-Giomi y Sun, 2016), y que se presenta en el apartado 2.3. 


\subsection{Participantes}

Para esta investigación, se seleccionan tres familias participantes. Debido al compromiso que supone recoger datos mensuales por un período de tiempo relativamente largo y constante (al menos un día entero) y la incomodidad de algunos miembros de la familia al mostrar el quehacer diario y las conversaciones íntimas, finalmente, las familias participantes mantienen cierta relación de confianza con la investigadora principal.

Por lo que concierne a características de las familias, por una parte, las tres son de cuatro miembros. Además del padre y la madre, están formadas por: 1) una niña de cinco años y un niño de 24 meses; 2) un niño de seis años y una niña de 36 meses, 3) un niño de cinco años y una niña de 28 meses. Por otra parte, las tres familias tienen perfiles similares: a) no viven en la gran ciudad, pero sí en un centro urbano; b) su nivel socioeconómico es medio-alto; c) tienen interés por la educación musical de sus hijos, ya que los llevan a la escuela de música como actividad extraescolar; d) al menos uno de los progenitores tiene conocimientos musicales; e) ambos adultos tienen estudios superiores, y f) la lengua materna es el catalán.

\subsection{Instrumentos de recogida de datos}

Los datos fueron recogidos principalmente con la grabadora de audio DLP (Digital Language Processor), capaz de grabar los sonidos hasta tres metros de distancia del entorno de grabación y con una duración de hasta 16 horas. En la actualidad, se usa para recoger datos tanto en centros escolares (Duncan et al., 2020) como en el entorno familiar (Elmquist et al., 2021). El DLP es un aparato de la medida de un afinador musical que, en este caso, lleva el/la hermano/a menor en un chaleco de tela especialmente diseñado con un bolsillo a la altura del pecho y con un estampado atractivo para que sea divertido de llevar puesto.

Esta grabadora está asociada a un sistema de software llamado LENA® (Language ENvironment Analysis), que es imprescindible para poder recoger los datos del aparato. Cada vez que se utiliza un DLP es necesario procesarlo con este programa informático para obtener la grabación de audio de ese día. Este software, muy utilizado para el estudio del desarrollo del habla, facilita cierta información de forma automática, por ejemplo, el número de palabras que se utilizan en el día concreto o el número de veces que interviene cada miembro de la familia. Por lo que concierne al análisis del contexto musical, que es el que interesa para esta investigación, el software no lo facilita. No obstante, la discreción, la calidad y la duración de la grabación, así como la captación de sonidos hasta tres metros de distancia de quien lleva el dispositivo, han sido imprescindibles para poder recoger los datos para este estudio.

Las grabaciones se realizaron en días festivos, en los que los/as niños/as no tenían que ir al colegio y pasaban más horas en el contexto familiar. La grabadora se activó en el momento en

@ Marta Dosaiguas Canal y Jèssica Pérez Moreno. The content of this article is the sole responsibility of the authors. The Revista Electrónica de LEEME and Universitat de València are not liable for any legal actions that may arise involving the article's content. Revista Electrónica de LEEME - Lista Electrónica Europea de Música en la Educación. http://ojs.uv.es/index/php/LEEME/index ISSN: 1575-9563. Editores: Universidad de Valencia y Jesús Tejada. Visibilidad de esta revista: SCOPUS, Emerging Sources Citation Index (Clarivate), EBSCO, CINDOC (CSIC), Citefactor, COPAC, Dialnet, DICE (CSIC), DOAJ, e-revistas (CSIC), EBSCO Premier, ERIH+, Gale Cengage Learning, IN-RECS, IRESIE, LATINDEX, MIAR, OCLC Worldcat, RESH, REDIB, RILM Core Journals, SUDOC, ULRICHS. Esta revista es de acceso libre mediante licencia Creative Commons 4.0 CC by. Política de archivado: etiqueta verde SHERPA-ROMEO. 
que el hermano/a menor se levantaba y hasta que se dormía por la noche, por lo que son grabaciones con una duración media de unas 14 horas.

Con el objetivo de conocer el contexto de cada grabación, se pidió a las familias participantes que, al finalizar el día en que se usó el DLP, enviasen a la investigadora principal una nota de audio con lo sucedido durante ese día tanto a nivel descriptivo - hora en que se despertaron los/as niños/as, lugares a los que acudieron, personas con las que compartieron el día - como a nivel interpretativo — cómo estaba el/la niño/a ese día, cómo se encontraban el padre y la madre y cómo se desarrolló la jornada-. El motivo de usar la nota de audio fue para facilitar la tarea a las familias sugiriendo una manera que pudiese ser inmediata para poder realizar el reporte en el mismo día de lo acontecido.

De la primera familia, se cuenta con 11 grabaciones; de la segunda familia, se cuenta con 2 y de la tercera tan solo con 1. El número de grabaciones se realizó en función de la disponibilidad de las familias y de la accesibilidad a las grabadoras. En total, se dispone de 190 horas de grabación.

\subsection{Procedimiento}

Una vez obtenidos los archivos de audio, se clasificaron según la familia de procedencia y el día en que fueron recogidos, acompañándolos en la misma carpeta del audio de contexto enviado por la familia. El análisis de cada archivo se inicia mediante la escucha de todo el audio, mientras se completa cada 30 segundos de audio una tabla validada por el equipo de investigación de la Universidad Estatal de Ohio (OSU, por sus siglas en inglés) (Costa-Giomi y Sun, 2016) (Tabla 1).

Tabla 1. Ejemplo primera escucha

\begin{tabular}{cccl}
\hline Tiempo & $\begin{array}{c}\text { Música } \\
\text { sí/no }\end{array}$ & $\begin{array}{c}\text { Distancia } \\
\text { del niño/de } \\
\text { la niña }\end{array}$ & Quién/qué está cantando/tocando/produciendo música \\
\hline 00:46:30 & Sí & Cerca & $\begin{array}{l}\text { Padre canta Puja al meu coet, de Pot Petit } \\
\text { 0adre canta y enciende en el reproductor Puja al meu coet, de Pot } \\
\text { Petit }\end{array}$ \\
\hline
\end{tabular}

En esta investigación, se ha considerado episodio musical aquel fragmento de audio en el que se escuchan sonidos organizados, ya sean producidos vocalmente o con percusión corporal, con un juguete, con un instrumento musical o con un reproductor de música digital. También se han considerado episodios musicales los sonidos producidos a través de la exploración vocal o durante el juego de los más pequeños (Pérez-Moreno y Viladot, 2016). Por otra parte, se considera una interacción musical válida aquella en la que: a) intervienen las voces de dos hermano/as; b) se escucha al menos una respuesta por parte de uno de los/as hermanos/as, y c) son calificados como musicales, dado que hay una organización sonora, algún elemento musical, algún ritmo o 
altura que se puede diferenciar del lenguaje hablado. Con estos criterios, se pudieron listar 58 interacciones entre hermanos; 25 interacciones de la familia A; 28 interacciones de la familia $\mathrm{B}$, y cinco interacciones de la familia C. Las interacciones recopiladas tienen un promedio de 44 segundos de duración.

Para responder a los objetivos del estudio, se añaden dos columnas más a la tabla de análisis propuesta por el equipo de OSU para registrar la información respecto de si se producen interacciones musicales y sobre el contexto de estas — lugar donde se producen-.

A continuación, se definen las dimensiones de análisis. Las dos primeras dan respuesta al primer objetivo específico de esta investigación: conocer las características específicas de este tipo de interacciones:

1) Orden de participación: ¿Quién inicia y finaliza la interacción? El objetivo es conocer quién propicia la interacción y quién la desarrolla, ya que puede ser un hermano u otro o bien una persona externa.

2) Lugar: ¿Dónde se realiza la acción? La finalidad es conocer el espacio físico en el que ocurren.

Las dos últimas dimensiones dan respuesta al objetivo específico de conocer las características musicales de estas interacciones:

3) Tipo de intervención: ¿Qué formato tiene la participación? El objetivo es constatar las características morfológicas de la interacción.

4) Fuente: ¿En qué naturaleza musical se basan? La finalidad es conocer la procedencia, el origen musical, de la construcción musical.

Puede ser que ese encuentro musical tenga como punto de partida una canción conocida por el investigador, se desarrolle a partir de una palabra entonada o se trate de una improvisación musical o de una melodía con una formalidad musical que respeta los cánones de composición, pero que ni el investigador ni las familias son capaces de reconocer ${ }^{1}$.

Una vez construido el instrumento de análisis, se procede a su validación mediante una prueba de jueces con tres expertos. Dos de los expertos pertenecen al área de educación musical, una de ellas específicamente de Educación Infantil y otro especializado en canto y dirección coral, mientras que la tercera experta es del área de salud vocal. Se compararon las observaciones de los jueces en referencia a tres fragmentos de los 58 por analizar, escogidos al azar. Se aplicó la fórmula de la confiabilidad individual para diferentes codificadores (Hernández et al., 2006 [citado por Blanch, 2010]), donde la confiabilidad individual es igual al número de unidades de

\footnotetext{
${ }^{1} \mathrm{Si}$ se trata de una melodía no reconocida por el investigador, se ha enviado el episodio musical a los padres y estos han decidido si se trataba de una canción conocida previamente o de una melodía de nueva creación. 
análisis codificadas correctamente por el codificador entre el número total de unidades de análisis. Con este proceso se obtuvo una versión definitiva del instrumento de análisis (Tabla 2).

Tabla 2. Dimensiones y categorías

\begin{tabular}{|c|c|c|c|c|}
\hline Dimensiones & Categoría & Descripción categoría & Subcategoría & $\begin{array}{l}\text { Descripción } \\
\text { subcategoría }\end{array}$ \\
\hline \multirow[t]{6}{*}{$\begin{array}{l}\text { Orden de } \\
\text { participación }\end{array}$} & Empieza & $\begin{array}{l}\text { Quién produce la primera } \\
\text { intervención musical }\end{array}$ & Hermano/a mayor & - \\
\hline & & & Hermano/a menor & - \\
\hline & & & Externo & $\begin{array}{l}\text { Cualquier persona u } \\
\text { objeto sonoro que no } \\
\text { sea ninguno de los/as } \\
\text { hermanos/a }\end{array}$ \\
\hline & Termina & $\begin{array}{l}\text { Quién es el último que } \\
\text { responde, antes de que haya } \\
\text { silencio o se cambie de } \\
\text { tema }\end{array}$ & Hermano/a mayor & - \\
\hline & & & Hermano/a menor & - \\
\hline & & & Externo & $\begin{array}{l}\text { Cualquier persona } \mathrm{u} \\
\text { objeto sonoro que no } \\
\text { sea ninguno de los/as } \\
\text { hermanos/a }\end{array}$ \\
\hline \multirow[t]{4}{*}{ Lugar } & Dónde & $\begin{array}{l}\text { Lugar de producción de la } \\
\text { intervención }\end{array}$ & $\begin{array}{l}\text { Interior (casa } \\
\text { propia) }\end{array}$ & Vivienda habitual \\
\hline & & & $\begin{array}{l}\text { Interior (no casa } \\
\text { propia) }\end{array}$ & $\begin{array}{l}\text { Lugar cerrado que no } \\
\text { sea vivienda habitual }\end{array}$ \\
\hline & & & Exterior & Lugar abierto \\
\hline & & & Coche & Vehículo privado \\
\hline
\end{tabular}

\begin{tabular}{|c|c|c|}
\hline \multirow[t]{4}{*}{$\begin{array}{l}\text { Tipo de } \\
\text { intervención }\end{array}$} & Imitación & $\begin{array}{l}\text { Repetición del motivo } \\
\text { musical, aunque se puedan } \\
\text { apreciar pequeñas } \\
\text { modificaciones }\end{array}$ \\
\hline & Diálogo & $\begin{array}{l}\text { Respuesta musical } \\
\text { consecuente }\end{array}$ \\
\hline & Contraste & $\begin{array}{l}\text { Respuesta musical no } \\
\text { consecuente con la pregunta }\end{array}$ \\
\hline & Sincronía & $\begin{array}{l}\text { Los/as dos hermanos/as } \\
\text { producen música en } \\
\text { paralelo }\end{array}$ \\
\hline \multirow[t]{4}{*}{ Fuente } & Canción & $\begin{array}{l}\text { Momento musical basado } \\
\text { en una canción reconocida } \\
\text { por el investigador y por los } \\
\text { padres* }\end{array}$ \\
\hline & $\begin{array}{l}\text { Palabra } \\
\text { entonada }\end{array}$ & $\begin{array}{l}\text { Momento musical basado } \\
\text { en la entonación concreta de } \\
\text { una palabra }\end{array}$ \\
\hline & $\begin{array}{l}\text { Improvisa } \\
\text { ción }\end{array}$ & $\begin{array}{l}\text { Momento musical basado } \\
\text { en la improvisación }\end{array}$ \\
\hline & Melodía & $\begin{array}{l}\text { Melodía con sentido } \\
\text { musical no reconocida por } \\
\text { el investigador }\end{array}$ \\
\hline
\end{tabular}


A modo de ejemplo de aplicación del instrumento, se muestra el análisis correspondiente a la interacción 32, que se ve transcrita en el primer apartado de los resultados (Tabla 3).

Tabla 3. Ejemplo de análisis (interacción 32, familia B, audio 1, 09:59:34 - 09:59:45)

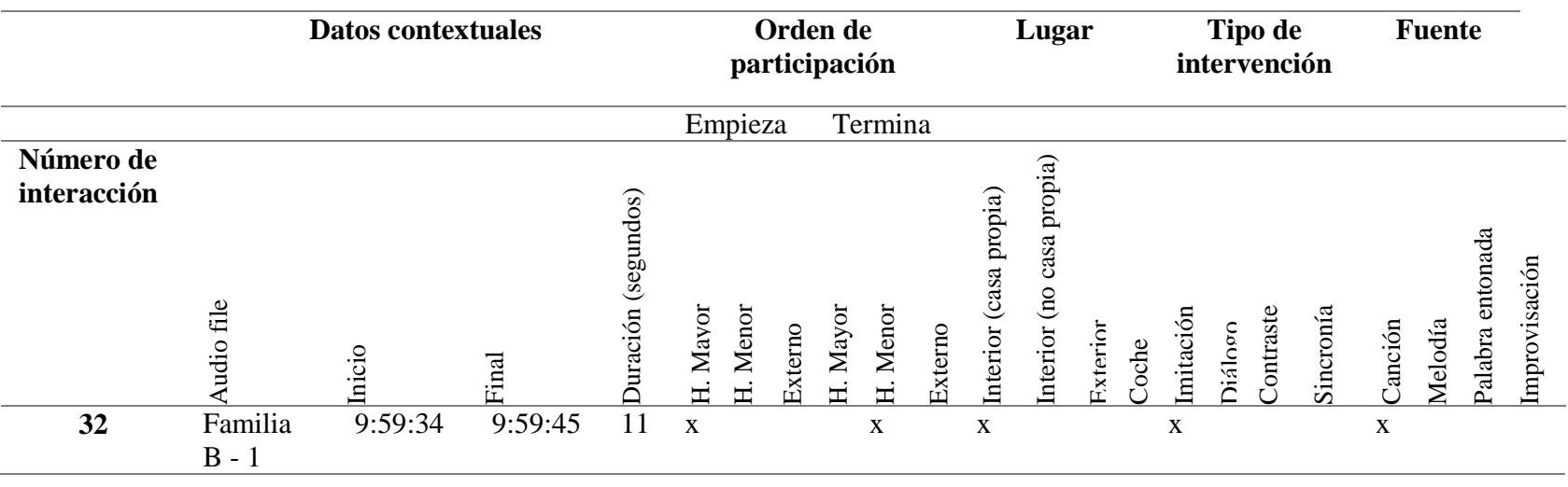

\section{Discusión de resultados}

Como se observa a partir del instrumento de análisis, cada audio es analizado en profundidad después de un número repetido de escuchas. En total, se identifican 61 episodios de interacción musical en los que participan los/as hermanos/a y, de estos, 58 corresponden a interacciones musicales entre sí, donde otras personas pueden estar presentes, pero la interacción entre hermanos/as se produce claramente. El interés de este artículo se centra en explicar los resultados que se refieren a las 58 interacciones entre hermanos/as, de las que se presentan datos referentes a las cuatro dimensiones estudiadas: orden de participación, lugar, tipo de intervención y fuente.

A pesar de que el tratamiento de los datos va a ser eminentemente cuantitativo, empezamos con un ejemplo que ayuda a captar la esencia de los datos analizados. En la figura 1, se muestra la transcripción de una de las interacciones, donde el hermano mayor canta una canción y, después, el menor la repite.

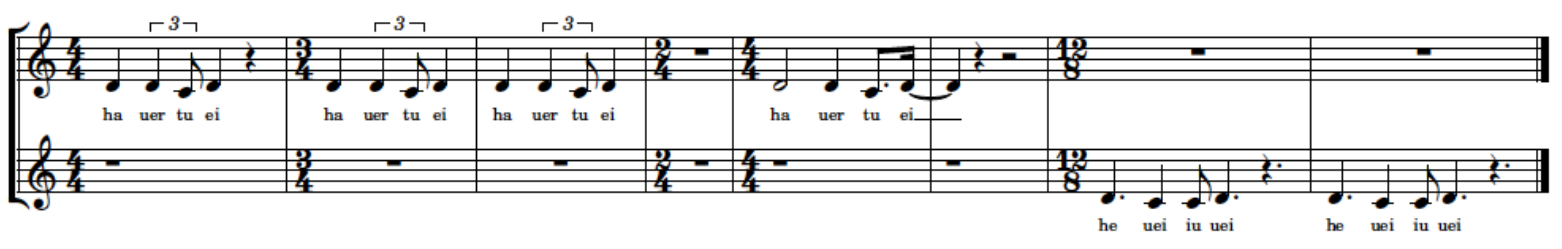

Figura 1. Ejemplo de interacción (interacción 32, familia B, audio 1, 09:59:34 - 09:59:45) 
En esta interacción se aprecia una imitación del motivo musical que el hermano mayor, David, ${ }^{2}$ canta. El motivo, aunque con un texto inventado, es de Highway to Hell, canción del grupo de rock AC/DC publicada en el año 1978. David ha cantado ya este motivo unas horas antes y ahora lo canta de nuevo; primero, modificando el ritmo y sin dejar los silencios de la canción original entre motivos; después, se detiene y lo canta una vez en su versión original (compás cinco). Seguidamente, la hermana menor, Mar, repite el motivo musical. Su forma de repetirlo es con alguna modificación tanto rítmica como melódica, pero se puede escuchar que es una variación del mismo motivo.

Por lo tanto, a partir de su análisis y en referencia a la participación (tabla 3), sabemos que quien inicia la interacción en este caso es el hermano mayor, David, y que es Mar, la hermana menor, quien la termina. Esta es una práctica habitual: los datos revelan que quien más invitaciones lanza a la interacción acostumbra a ser el mayor de los hermanos; concretamente, se constata que es quien la empieza en la mayoría de los encuentros $(n=38)$, un 65,5\% de las veces. Contrariamente, es el hermano menor quien termina la interacción mayoritariamente $(n=37)$, un $63,8 \%$ de las ocasiones (Tabla 4 ).

Tabla 4. Cuantificación de la participación respecto a inicio y final en las interacciones

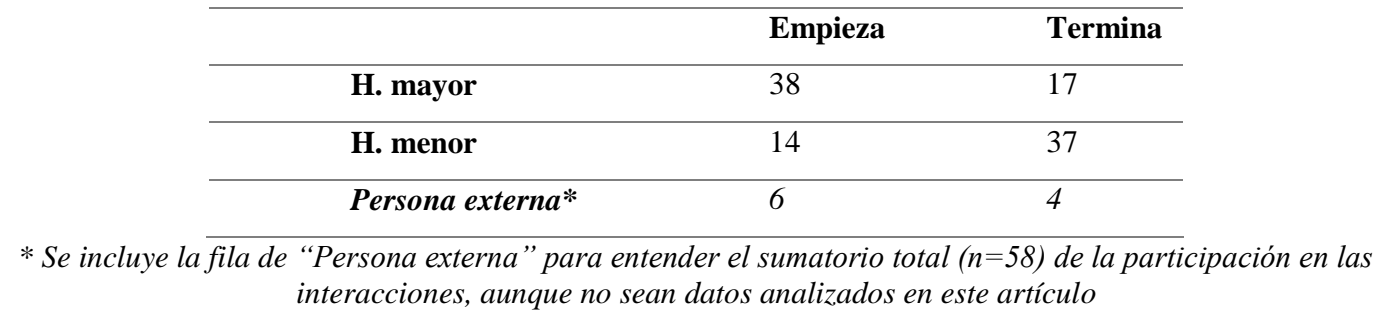

Como ya apuntaron estudios previos (Barret, 2009; Gingras, 2012; Koops, 2014, 2017; $\mathrm{Wu}, 2018$ ), las interacciones musicales fraternales funcionan con los roles de la teoría socioconstructivista: miembro experto/inexperto. Por tanto, acostumbra a ser el/la hermano/a mayor, el miembro experto musicalmente, quien inicia la acción musical que conduce a la intervención del hermano pequeño. Este hecho puede ser interpretado básicamente en dos direcciones: por una parte, puede ser que el/la hermano/a mayor se exprese musicalmente sin esperar participar de una interacción con el/la menor, que se muestra atento y mayormente lo imita con ganas de participar musicalmente sin obtener una respuesta de vuelta. Por otra parte, podría deberse también a una interacción intencionada por parte del/de la mayor, que ofrece repertorio y/o ideas musicales que el/la menor es capaz de liderar a continuación. A modo de ejemplo, en una interacción de la familia C, María, la hermana menor, está cantando una canción. El hermano mayor, Nico, empieza otro tipo de sonido improvisado e inmediatamente María para

\footnotetext{
${ }^{2}$ Se han utilizado seudónimos para mantener el anonimato de las personas participantes. 
de cantar y repite el sonido de Nico. Nico no responde a su hermana. Vemos aquí, de nuevo, cómo el hermano mayor es un referente para María.

En cuanto a la dimensión de lugar, se puede afirmar que el interior del hogar es el sitio donde se han producido más interacciones; de hecho, casi 3/4 partes de ellas $(74,2 \%)$. Este es el caso de la interacción de la Figura 1. El resto de interacciones tuvieron lugar, por orden de mayor a menor incidencia: en el exterior, al aire libre (10,3\%), seguido muy de cerca por el coche $(8,6 \%) \mathrm{y}$, finalmente, por espacios interiores que no son el propio hogar, como pueden ser, por ejemplo, un comercio o vivienda que no es de la familia $(5,2 \%)$.

Teniendo en cuenta que los datos se recogieron en fin de semana, no sorprende constatar que el gran volumen de encuentros musicales fraternales tuvo lugar en casa. Es relevante mostrar que, en las pocas ocasiones que comparten dentro de un coche, también se producen interacciones musicales muy ricas. Este espacio es definido por muchos autores como un lugar familiar con la particularidad de tener espacio propio e íntimo para ellos/as. El hecho de estar en la parte trasera del coche viendo a los progenitores, pero sin ser vistos, desencadena distintas actividades musicales con distintos formatos de participación familiar (Barrett, 2009; de Vries, 2009; Dosaiguas, et al., 2021; Koops, 2014; Lamont, 2008; Lum, 2009; Wilfinger, et al., 2011).

Por lo que concierne al tipo de intervención, la mayoría de las veces, cada interacción se mantiene en la misma tipología de inicio a fin, como en el ejemplo de la Figura 1. Sin embargo, en siete fragmentos se ha detectado más de un formato e, incluso, tres tipos distintos en un mismo fragmento (por ejemplo, empiezan imitando y acaban en sincronía). En esta dimensión ( $\mathrm{n}=70)$, la tipología protagonista ha sido la imitación, identificada en 29 ocasiones, lo que representa el $41 \%$ del total. Estos resultados coinciden con lo que indica la literatura existente, donde se muestra la imitación de sonidos y música como la forma de interacción musical por excelencia en la primera infancia (Malloch y Trevarthen, 2009; Gordon, 2013).

En concordancia con el estudio de Cirelli et al. (2020), los resultados de esta investigación demuestran que la imitación musical del material ofrecido por el/la hermano/a mayor es un tipo de intervención recurrente, donde este marca una dirección que sigue el/la menor. Se ha visto que los/as pequeños/as responden a la idea musical que el/la mayor ha propuesto, es decir, que el/la mayor inicia la propuesta y el/la menor la termina (véase el ejemplo de la Figura 2). Esta asociación de roles destaca el deseo del/de la menor de participar en la acción musical, de incluirse y desarrollarse a partir de la invitación facilitada. 


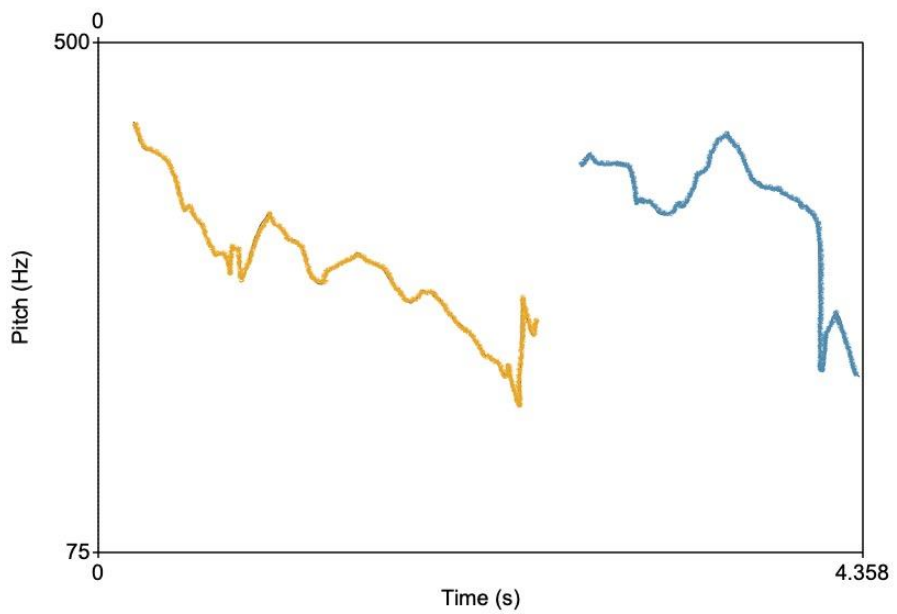

Figura 2. Ejemplo de imitación entre hermano/a mayor (naranja) y menor (azul) (interacción 20, familia A, audio 9,00:15:18 - 00:15:22)

De acuerdo con el estudio de Wu (2018), que remarca la importancia de la influencia entre hermanos/as, este estudio permite constatar también cómo las interacciones musicales en sincronía, entendidas como la producción simultánea de sonidos musicales (como, por ejemplo, cantar juntos), son de especial relevancia por el hecho de estar en un mismo momento haciendo música juntos. Leimbrink (2009) estableció dos secuencias de interacción preverbal: la sincronía y la alternancia. La primera es mayoritaria: concretamente, se ha identificado sincronía en 20 ocasiones, lo que representa el $29 \%$ de las interacciones. La comunicación por turnos - que se ha categorizado como diálogo - y el contraste — categorizado cuando no se identifica relación entre los sonidos musicales que se están produciendo simultáneamente- han resultado ser también una forma de interacción, aunque menos abundante en los datos analizados (10 ocasiones $-14 \%$ - y 11 ocasiones $-16 \%$, respectivamente).

Respecto a la dimensión fuente $(\mathrm{n}=70)$, los resultados indican que la protagonista es la canción (base de la interacción en 28 ocasiones - 40 \%-). La gran cantidad de canciones que se escuchan en estas grabaciones ayudan a entender cómo se está produciendo la enculturación de estos niños y niñas (Campbell, 2010). La música está presente en su cotidianidad y están expuestos a repertorios muy variados, desde canciones de cuna hasta el rock o el reggaetón. La canción, ya sea cantada en directo por distintos miembros de la familia o por otras personas con las que comparten el día o bien recibida a través de distintos dispositivos electrónicos, es la fuente musical con más presencia en las grabaciones. Dado que, como se ha visto, la imitación es una forma de interacción muy potente, no es de extrañar que una actividad musical recurrente sea aprender por imitación y cantar este repertorio al que se está expuesto.

Los niños y niñas del estudio también interaccionan improvisando música (concretamente en 27 ocasiones - 39\%-). Se constata que se expresan libremente sin necesidad de reproducir 
la música que conocen con la misma facilidad y frecuencia que en la categoría anterior. Estos resultados apoyan la musicalidad innata, el potencial para la comunicación musical con el que todos nacemos (Malloch y Trevarthen, 2009).

\section{Conclusiones}

Tal y como afirman Koops y Kuebel (2019), las relaciones entre hermanos/as influyen en su desarrollo musical. Los resultados de este estudio muestran que las interacciones musicales fraternales son una actividad propia de la cotidianidad de una familia y que pone en relieve el carácter social de la música, la cual genera deseo de participación. Según Papoušek (1996, p.38), "las contribuciones más relevantes del desarrollo comunicativo y de la integración de capacidades en los niños ocurre en las intervenciones educativas no-conscientes". La principal contribución de este estudio es visibilizar las interacciones musicales entre hermanos/as como actividad cotidiana desarrollada en un contexto no formal y definir unas dimensiones de estudio para conocer las características de las mismas: orden de participación, quién empieza y termina; lugar, dónde se producen; tipo de intervención, en calidad de construcción del sonido - repetición, imitación, contraste o sintonía-, y fuente, si proviene de un material musical previo o es de nueva creación.

Estudios previos ya exponían que en un día se producen intervenciones musicales cortas entre diferentes miembros de la familia en gran cantidad de ocasiones. Por ejemplo, Benetti (2017) analiza el audio de un día entero de un bebé; Dean (2011) observó a su hijo durante 5 días enteros, y Gingras (2012) a través de cuestionarios y entrevistas de 60 minutos analizó a 5 familias. En contraste con estos estudios, esta investigación destaca por haber analizado 14 días enteros (que corresponden a 190 horas de grabación) de 3 familias distintas en las que se han detectado todos los momentos musicales y se ha analizado nuestro foco de interés: las interacciones musicales entre hermanos. Dentro del destacado volumen de interacciones musicales entre los diferentes miembros de la familia que se identifican en este estudio, por lo que respecta a hermanos/as, el volumen es discreto (se puede consultar más datos en Dosaiguas et al., 2021). El proceso de análisis ha sido de especial dificultad por el formato de audio de la información obtenida. Definir si las interacciones eran musicales o no ha sido complejo porque la frontera entre palabra entonada y canto es muy borrosa en algunos casos. Como sabemos, pasar de la expresividad hablada al canto es una acción recurrente en los más pequeños. La expresividad sonora de la entonación permite la exploración vocal del niño y la niña y muchas veces una entonación deriva a un momento musical (Pérez-Moreno, 2017). Estos casos han precisado de numerosas escuchas para decidir si categorizar ese episodio como musical.

Otro resultado de este estudio que coincide con lo expuesto en la literatura es que los niños y niñas occidentales acostumbran a improvisar vocalmente. Según Adachi (2013), el canto espontáneo de los niños y niñas japoneses en la etapa preescolar es diferente al canto espontáneo 
investigado en Occidente, en el sentido de que estos japoneses se muestran menos creativos y repiten canciones o melodías que ya han escuchado, al contrario de lo que pasa en Occidente. En el presente estudio, donde los niños y niñas suelen improvisar y cantar, creando en el mismo momento y con la misma facilidad y regularidad. Como prospectiva sería interesante, por una parte, realizar un seguimiento de estas actividades en niños/as de mayor edad para ver cuál es la relación entre improvisación y reproducción de una canción a lo largo de su desarrollo musical y ver si este equilibrio se mantiene en sus expresiones musicales. Por otra, estudiar posibles diferencias relacionadas con la cultura. Así mismo, es necesario seguir indagando en la relación entre el desarrollo del lenguaje hablado y la expresión musical de los pequeños para ver qué tipo de interacciones musicales se establece con hermanos/as pequeños/as que aún no han desarrollado el lenguaje verbal.

Una de las dificultades del estudio es el número de familias participantes. Algunas de las razones de esta limitación responden a: 1) la dificultad de encontrar familias que cumplan con los criterios de selección y que permitan el acceso a su intimidad y se comprometan a una recogida de datos regular en el tiempo; 2) la gestión del volumen de datos por una sola investigadora - teniendo en cuenta que cada grabación es de alrededor de 14 horas de duración-, y 3) la obtención final de los datos — se necesita disponer del software LENA® para extraerla información de audio-.

Otra de las limitaciones, pero esta vez del propio instrumento de recogida de datos, es la unimodalidad de este, que responde exclusivamente a la obtención de datos sonoros. Esta restricción de tipología de los datos presenta otra cara llena de potencialidades, como la recogida de datos no intrusiva - la discreción y la ligereza del dispositivo permiten la grabación continua y este recoge datos de todo el día de los pequeños- y el análisis imparcial o neutro, no sujeto a la percepción de investigadores ni progenitores. Cabe destacar la diferencia entre lo que los progenitores explican a través de los audios de voz que envían después de apagar el dispositivo el día de grabación con el resultado de los datos analizados. Por poner un ejemplo, una de las familias comenta que ese día no pasó nada interesante musicalmente hablando y en cambio se descubre que ha habido música durante seis horas ese día.

El presente estudio muestra el paisaje sonoro de días enteros en un entorno familiar de las características descritas anteriormente y representa un paso adelante en el estudio de la música en la vida cotidiana, específicamente de las interacciones musicales entre hermanos. Animamos a más investigadores a adentrarse en esta díada para seguir avanzando en el estudio de la educación musical en la etapa Infantil.

\section{Financiación y agradecimientos}

Este trabajo ha sido realizado en el marco del programa de doctorado en Educación de la Universidad Autónoma de Barcelona. No se ha recibido un financiamiento específico, pero queremos agradecer a la Dra. Costa-Giomi de la Universidad Estatal de Ohio la cesión de los dispositivos DLP para la realización de este estudio. 


\section{Referencias}

Adachi, M. (2013). The nature of music nurturing in Japanese preschools. En P. Campbell y T. Wiggins (Eds.), The Oxford handbook of children's musical cultures (pp.449-465). Oxford University Press.

Barrett, M.S. (2009). Sounding lives in and through music: a narrative inquiry of the 'everyday' musical engagement of a young child. Journal of Early Childhood Research, 7(2), 115134. https://doi.org/10.1177/1476718X09102645

Barrett, M. y Stauffer, S. (Eds.) (2012). Narrative soundings: an anthology of narrative inquiry in music education. Springer.

Blacking, J. (2006). ¿Hay música en el hombre? Alianza Editorial.

Blanch, S. (2010). Expectatives parentals i pràctiques socioeducatives familiars. Influència mútua. (Tesis doctoral). Universitat Autònoma de Barcelona. https://www.tdx.cat/handle/10803/4781

Benetti, L. (2017). Infant vocal imitation of music. (Tesis doctoral). The Ohio State University. https://etd.ohiolink.edu/

Bordons, G. y Casals, A. (2012). Poesia, música i escola: un triangle sonor. Temps d'Educació, 42, 11-30. https://raco.cat/index.php/TempsEducacio/article/view/259111/346403

Brandt, A., Gebrian, M. y Slevc, L.R. (2012). Music and early language acquisition. Frontiers in Psychology, 3(SEP), 1-17. https://doi.org/10.3389/fpsyg.2012.00327

Brodsky, W. Sulkin, I. y Hefer, M. (2020). Musical engagement among families with young children: a CMBI (V.972) study. Early Child Development and Care, 190(9), 1483-1496. https://doi.org/10.1080/03004430.2019.1671834

Campbell, P.S. (2010). Musical enculturation: sociocultural influences and meanings of children's experiences in and through music. En M.S. Barrett (Ed.), A cultural psychology of music education (pp.61-81). Oxford University Press.

Casals, A. (2017). La música como práctica social y vivencia cultural. En C. Gluschankof y J. Pérez-Moreno (Eds.), La música en educación infantil: investigación y práctica (pp.1729). Dairea.

Cirelli, L.K., Peiris, R., Tavassoli, N., Recchia, H. y Ross, H. (2020). It takes two to tango: Preschool siblings' musical play and prosociality in the home. Social Development, 29(4), 964-975. https://doi.org/10.1111/sode.12439 
Connidis, I.A., y Campbell, L.D. (1995). Closeness, confiding, and contact among siblings in middle and late adulthood. Journal of Family Issues, 16(6), 722745. https://doi.org/10.1177/019251395016006003

Costa-Giomi, E. y Benetti, L. (2017). Through a baby's ears: Musical interactions in a family community. International Journal of Community Music, 10(3), 289-303. https://doi.org/10.1386/ijcm.10.3.289 1

Costa-Giomi, E. y Sun, X. (2016). Infants' soundscapes: A day in the life of a family. En J. Bugos (Ed.), Contemporary research in music learning: a lifespan perspective, (pp.87-96). Routledge.

De Vries, P. (2006). Being there: creating music-making opportunities in a childcare centre. International Journal of Music Education,24(3), 255-270. https://doi.org/10.1177/0255761406069661

De Vries, P. (2009). Music at home with the under fives: what is happening? Early Child Development and Care, 179(4), 395-405. https://doi.org/10.1080/03004430802691914

Dean, B. (2011). Oscar's music: A descriptive study of one three year-old's spontaneous music -making at home. (Tesis doctoral no publicada). Birmingham City University. https://d1wqtxts1xzle7.cloudfront.net/

Dean, B. (2019). Spontaneous singing and musical agency in the everyday home lives of three and four-year-old children. En S. Young y B. Ilari (Eds.), Music in early childhood: Multi-disciplinary perspectives and inter-disciplinary exchanges (pp.103-118). Springer.

Dosaiguas, M. (2021). ¿Qué sabemos sobre la interacción musical entre hermanos? Revisión de estudios sobre la música en el entorno familiar. En J.A. Marín, J.M. Trujillo, G. Gómez y M.N. Campos (Eds.), Hacia un modelo de investigación sostenible en educación (pp.234-250). Dykinson. https://ebin.pub/hacia-un-modelo-de-investigacion-sostenible

Dosaiguas, M., Pérez-Moreno, J., Gluschankof, C. y Costa-Giomi, E. (2021). Listening to a siblings' day: musical interactions in a family setting. Early Child Development and Care, 191(12), 1843-1857. https://doi.org/10.1080/03004430.2020.1863392

Duncan, R.J., King, Y.A., Finders, J.K., Elicker, J., Schmitt, S.A., y Purpura, D.J. (2020). Prekindergarten classroom language environments and children's vocabulary skills. Journal of Experimental Child Psychology, 194, 104829. https://doi.org/10.1016/j.jecp.2020.104829

Dunn, J. (2007). Siblings and socialization. En J.E. Grusec y P.D. Hastings (Eds.), Handbook of socialization: theory and research (pp.309-327). Guilford Press.

@ Marta Dosaiguas Canal y Jèssica Pérez Moreno. The content of this article is the sole responsibility of the authors. The Revista Electrónica de LEEME and Universitat de València are not liable for any legal actions that may arise involving the article's content. Revista Electrónica de LEEME - Lista Electrónica Europea de Música en la Educación. http://ojs.uv.es/index/php/LEEME/index ISSN: 1575-9563. Editores: Universidad de Valencia y Jesús Tejada. Visibilidad de esta revista: SCOPUS, Emerging Sources Citation Index (Clarivate), EBSCO, CINDOC (CSIC), Citefactor, COPAC, Dialnet, DICE (CSIC), DOAJ, e-revistas (CSIC), EBSCO Premier, ERIH+, Gale Cengage Learning, IN-RECS, IRESIE, LATINDEX, MIAR, OCLC Worldcat, RESH, REDIB, RILM Core Journals, SUDOC, ULRICHS. Esta revista es de acceso libre mediante licencia Creative Commons $4.0 \mathrm{CC}$ by. Política de archivado: etiqueta verde SHERPA-ROMEO. 
Elmquist, M., Finestack, L., Kriese, A., Lease, E. y McConnell, S. (2021). Parent education to improve early language development: A preliminary evaluation of LENA StartTM. Journal of Child Language, 48(4), 670-698. https://doi.org/10.1017/S0305000920000458

Eriksen, S. y Gerstel, N. (2002). A labor of love or labor itself: Care work among adult brothers and sisters. Journal of Family Issues, 23(7), 836-856. https://doi.org/10.1177/019251302236597

Forrester, M.A. (2010). Emerging musicality during the pre-school years: A case study of one child. Psychology of Music, 38(2), 131-158. https://doi.org/10.1177/0305735609339452

Gingras, P. (2012). Music at Home: A Portrait of Family Music-Making. (Tesis doctoral no publicada). University of Rochester. https://urresearch.rochester.edu/file

Gordon, E. (2003). A music learning theory for newborn and young children. GIA Publications.

Howe, N., Ross, H.S. y Recchia, H. (2014). Sibling relations in early and middle childhood. En P.K. Smith y C.H. Hart (Eds.), Wiley Blackwell handbooks of developmental psychology. The Wiley Blackwell handbook of childhood social development (pp.356-372). WileyBlackwell.

Howe, N., Porta, S.D., Recchia, H. y Ross, H. (2016). Because if you don't put the top on, it will spill: A longitudinal study of sibling teaching in early childhood. Developmental Psychology, 52(11), 1832-1842. https://doi.org/10.1037/dev0000193

Koops, L.H. (2014). Songs from the car seat: Exploring the early childhood music-making place of the family vehicle. Journal of Research in Music Education, 62(1), 52-65. https://doi.org/10.1177/0022429413520007

Koops, L.H. (2017). The enjoyment cycle: A phenomenology of musical enjoyment of 4- to 7year-olds during musical play. Journal of Research in Music Education, 65(3), 360-380. https://doi.org/10.1177/0022429417716921

Koops, L.H. (2020). Parenting musically. Oxford University Press.

Koops, L.H. y Kuebel, Ch.R. (2019). Probing the dynamics of sibling interactions in relation to musical development. En S. Young y B. Ilari (Eds.), Music in early childhood: Multidisciplinary perspectives and inter-disciplinary exchanges (pp.39-58). Springer Verlag.

Lamont, A. (2008). Young children's musical worlds: musical engagement in 3.5-year olds. Journal of Early Childhood Research, 6(3), 247261. https://doi.org/10.1177/1476718X08094449 
Leimbrink, K. (2009). The vocal development in the first year of life: how do infants communicate? En A.R. Adessi y S. Young (Eds), Proceedings of the European Network of Music Educators and Researchers of Young Children (pp.423-429). Bononia University Press.

Lum, C.H. (2009). Musical memories: snapshots of a Chinese family in Singapore, Early Child Development and Care, 179(6), 707-716. https://doi.org/10.1080/03004430902944296

Malloch, S. y Trevarthen, C. (2009). Musicality: communicating the vitality and interests of life. En S. Malloch y C. Trevarthen (Eds.), Communicative musicality. Exploring the basis of human companionship (pp.1-11). Oxford University Press.

Norris, S. (2004). Analyzing multimodal interaction: a methodological framework. Routledge.

Papoušek, M. (1996) Intuitive parenting: a hidden source of musical stimulation in infancy. En I. Deliège y J. Sloboda (Eds.) Musical beginnings (pp.88-112). Oxford University Press.

Papoušek, M. y Papoušek, H. (1981). Musical elements in the infant's vocalizations: Their significance for communication, cognition and creativity. En L.P. Lipsitt (Ed.), Advances in Infancy Research (pp.163-224). Ablex.

Pérez-Moreno, J. (2017). La musicalidad comunicativa, fuente de las relaciones humanas. En C. Gluschankof y J. Pérez-Moreno (Eds), La música en educación infantil: investigación y práctica (pp.31-44). Dairea.

Pérez-Moreno, J. y Viladot, L. (2016). El juego musical infantil. Revista electrónica LEEME, 37, 51-62. https://ojs.uv.es/index.php/LEEME/article/view/9878/9296

Piaget, J. (1965). The stages of the intellectual development of the child. En B. Marlowe y A. Canestrari (Eds.), Educational psychology in context: Readings for future teachers (pp.98-106). Thousand Oaks, CA: Sage Publications.

Politimou, N., Stewart, L., Müllensiefen, D. y Franco, F (2018). Music@ Home: A novel instrument to assess the home musical environment in the early years. PLoS ONE, 13(4), 1-23. https://doi.org/10.1371/journal.pone.0193819

Prime, H., Pauker, S., Plamondon, A., Perlman, M. y Jenkins, J. (2014). Sibship size, sibling cognitive sensitivity, and children's receptive vocabulary. Pediatrics, 133(2), 394-401. https://doi.org/10.1542/peds.2012-2874

Schaal, N.K., Politimou, N., Franco, F., Stewart, L. y Müllensiefen, D. (2020). The German Music@ Home: Validation of a questionnaire measuring at home musical exposure and interaction of young children. PLoS ONE, 15(8), 1-15. https://doi.org/10.1371/journal.pone.0235923 
Small, C. (1999). El musicar: un ritual en el espacio social. TRANS-Revista Transcultural de Música, 4. http://www.sibetrans.com/trans/p11/trans-4-1999

Sole, M. (2014). Songs from de crib: toddlers' private bedtime vocalizations. A collective case study. (Tesis Doctoral no publicada). Columbia University. https://www.academia.edu/7819563/

Spitze, G.D. y Trent, K. (2018). Changes in individual sibling relationships in response to life events. Journal of Family Issues, 39(2), 503-526. https://doi.org/10.1177/0192513X16653431

Thanh, N.C. y Thanh, T.T. (2015). The interconnection between the interpretivism paradigm and qualitative methods in education. American Journal of Educational Science, 1(2), 24-27. http://files.aiscience.org/journal/article/pdf/70380008.pdf

Teti, D.M. (2001). Retrospect and prospect in the psychological study of sibling relationships. En J.P. McHale y W.S. Grolnick (Eds.), Retrospect and prospect in the psychological study of families (pp.193-224). Routledge.

VanWynsberghe, R. y Khan, S. (2007). Redefining case study. International Journal of Qualitative Methods, 6(2), 80-94. https://doi.org/1.1177/160940690700600208

Vilar, M. (2014). Acerca de la educación musical. Revista Electrónica de LEEME, 13, 1-23. https://ojs.uv.es/index.php/LEEME/article/view/9748/9182

Vigotsky, L.S. (1978). Mind in society: The development of higher psychological processes. Harvard University Press.

Weaver, S.E., Coleman, M. y Ganong, L.H. (2003). The sibling relationship in young adulthood: Sibling functions and relationship perceptions as influenced by sibling pair composition. $\begin{array}{lllll}\text { Journal of } & \text { Family } & \text { 245- }\end{array}$ 263. https://psycnet.apa.org/doi/10.1177/0192513X02250098

Wilfinger, D., Meschtscherjakov, A., Murer, M., Osswald, S. y Tscherligi, M. (2011). Are we there yet? A probing study to inform design for rear seat of family cars. En P. Campos, N. Graham, J. Jorge, N. Nune, P. Palanque y M. Winckler (Eds.), Proceedings from INTERACT 2011: Human-Computer Interaction, 13th International Conference Part II (pp.657-674). Springer.

Willis, J.W. (2007). Foundations of qualitative research: Interpretive and critical approaches. SAGE Publications, Inc. https://www.doi.org/10.4135/9781452230108

Wu, Y.T. (2018). Musical development of young children of the Chinese diaspora in London. (Tesis doctoral). UCL Institute of Education. https://discovery.ucl.ac.uk 


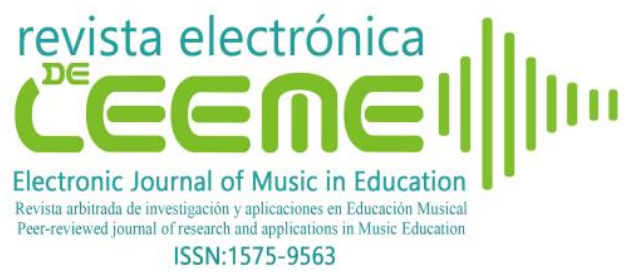

Marta Dosaiguas Canal y Jèssica Pérez Moreno Estudio exploratorio de las interacciones musicales entre hermanos/as en el entorno cotidiano (Revista Electrónica de LEEME) Número 48, pp. 39-58 https://ojs.uv.es/index.php/LEEME/index

ISSN:1575-9563

Young, S. (2016). Early childhood music education research: An overview. Research Studies in Music Education, 38(1), 9-21. https://doi.org/10.1177/1321103X16640106

@ Marta Dosaiguas Canal y Jèssica Pérez Moreno. The content of this article is the sole responsibility of the authors. The Revista Electrónica de LEEME and Universitat de València are not liable for any legal actions that may arise involving the article's content. Revista Electrónica de LEEME - Lista Electrónica Europea de Música en la Educación.http://ojs.uv.es/index/php/LEEME/index ISSN: 1575-9563. Editores: Universidad de Valencia y Jesús Tejada. Visibilidad de esta revista: SCOPUS, Emerging Sources Citation Index (Clarivate), EBSCO, CINDOC (CSIC), Citefactor, COPAC, Dialnet, DICE (CSIC), DOAJ, e-revistas (CSIC), EBSCO Premier, ERIH+, Gale Cengage Learning, IN-RECS, IRESIE, LATINDEX, MIAR, OCLC Worldcat, RESH, REDIB, RILM Core Journals, SUDOC, ULRICHS. Esta revista es de acceso libre mediante licencia Creative Commons 4.0 CC by. Política de archivado: etiqueta verde SHERPA-ROMEO. 